\title{
Editorial
}

\section{Information Geometry: From Black Holes to Condensed Matter Systems}

\author{
Tapobrata Sarkar, ${ }^{1}$ Hernando Quevedo, ${ }^{2}$ and Rong-Gen Cai ${ }^{3}$ \\ ${ }^{1}$ Department of Physics, Indian Institute of Technology, Kanpur 208016, India \\ ${ }^{2}$ Instituto de Ciencias Nucleares, Universidad Nacional Autonoma de Mexico, Apartado Postal 70-543, \\ 04510 Mexico City, DF, Mexico \\ ${ }^{3}$ Institute of Theoretical Physics, Chinese Academy of Sciences, Beijing 100190, China
}

Correspondence should be addressed to Tapobrata Sarkar; tapo@iitk.ac.in

Received 8 September 2013; Accepted 8 September 2013

Copyright (C) 2013 Tapobrata Sarkar et al. This is an open access article distributed under the Creative Commons Attribution License, which permits unrestricted use, distribution, and reproduction in any medium, provided the original work is properly cited.

The role of geometry in understanding physical phenomena, from large to small scales, has become an exciting arena of research in the recent past. Novel geometric insights into the phases of matter at different length scales indicate a deep connection between properties of dissimilar physical systems. The present issue contains original research articles aimed towards furthering our understanding of these aspects.

The paper "A cosmological scaling relation for describing the late time dynamics" explores the relation between quantum fluctuations and Cosmological dynamics. This is obtained via a proposed scaling that arises from black hole physics, between the mass and minimal information of a given system. The emergence of dark energy is shown to follow as a consequence of this scaling relation.

In the paper "On thermodynamics of charged and rotating asymptotically AdS black strings," the authors study the thermodynamics of charged, rotating black strings. This is done by treating the Cosmological constant as pressure and using its conjugate volume. Issues of stability of these systems is analyzed, and it is established that they are stable and that they do not undergo second order phase transitions.

In the paper "Two-dimensional Einstein manifolds in geometrothermodynamics," a Riemannian geometric analysis is carried out for a class of thermodynamic systems, for which the associated curvature is a constant. For a particular class of such systems, a differential equation scheme is set up, and a description of a polytropic fluid is obtained.
In "Legendre invariance and geometrothermodynamics description of the 3D charged-dilaton black hole," the authors explore a Legendre invariant thermodynamic formalism for charged dilatonic black holes. This is used to obtain the phase properties of such black holes. Issues of stability are also studied, and a comparative analysis of various geometric approaches is made.

In the paper "Geometrothermodynamics of Myers-Perry black holes," thermodynamics and its Legendre invariant formulation is studied for various cases in black holes of five dimensions. A three-dimensional parameter manifold arising out of such a construction is also discussed, which gives rise to divergences in addition to the ones appearing due to the phase structure of the theory, and a theoretical explanation for these is offered.

The paper "Geometric curvatures of plane symmetry black hole" studies the geometric structure of a class of black holes, in different formalisms. First- and second-order phase transitions are investigated, and the scalar curvatures calculated in these cases. Local thermodynamic stability is also discussed via the heat capacity.

In "Geometric description of the thermodynamics of the noncommutative Schwarzschild black hole," a Legendre invariant thermodynamic formulation of a class of noncommutative black holes is discussed. Via the geometric structure of the equilibrium state space, the authors analyze the phase structure of the system, by treating the noncommutativity parameter at par with other thermodynamic variables. 
This issue brings together a collection of research papers on the application of geometric methods to a wide variety of physical systems. We hope this will be a useful volume for researchers working in related areas.

Tapobrata Sarkar Hernando Quevedo Rong-Gen Cai 

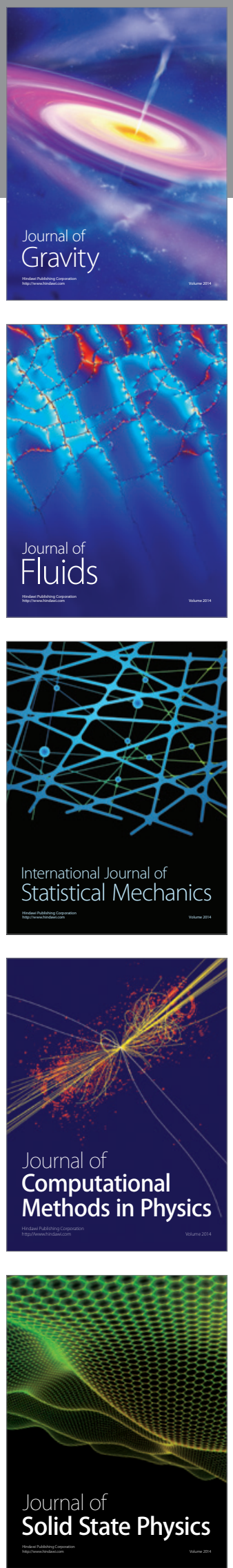

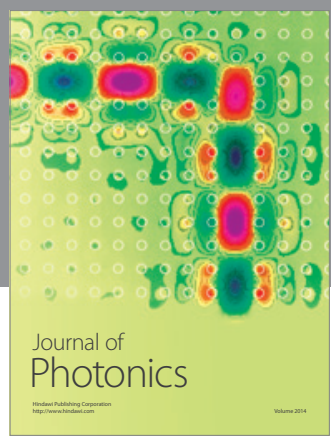

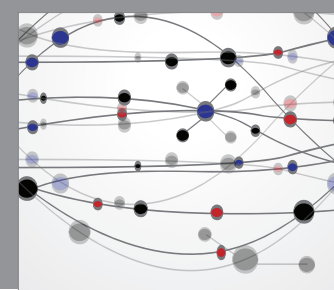

The Scientific World Journal

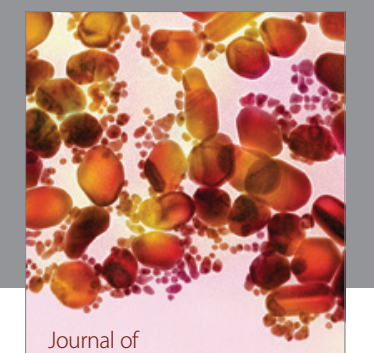

Soft Matter
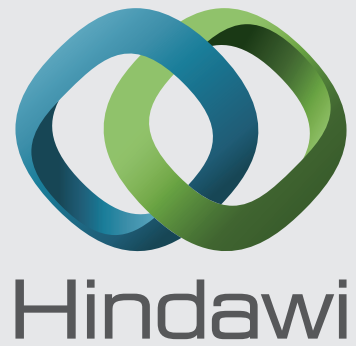

Submit your manuscripts at

http://www.hindawi.com
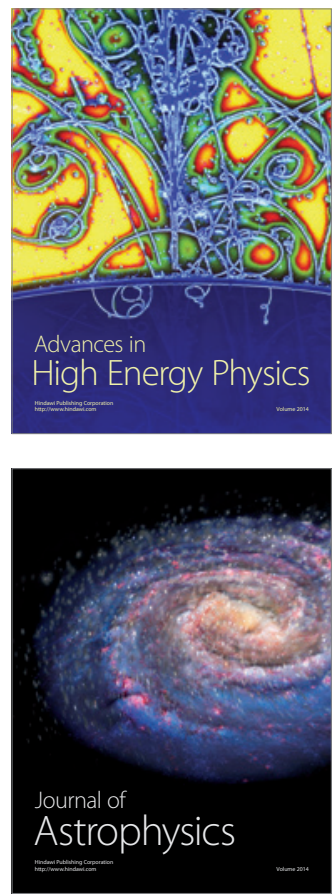
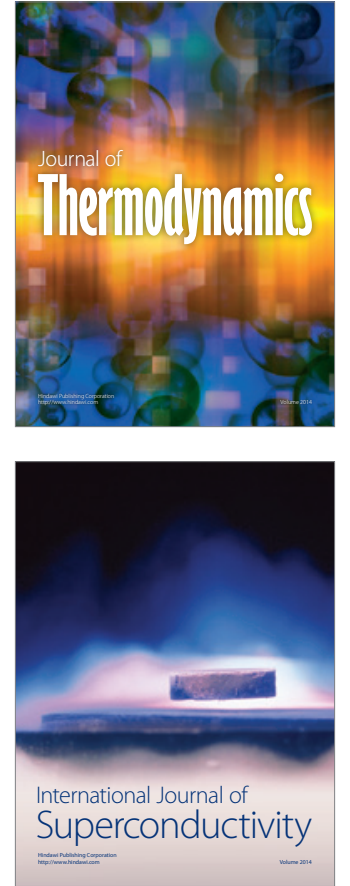
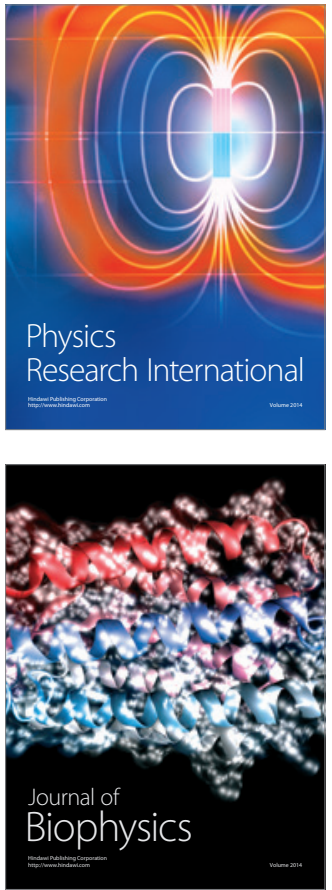
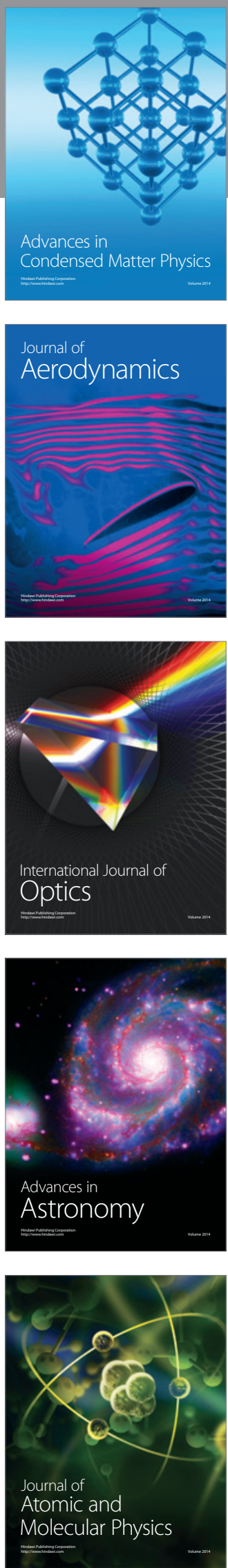